\title{
Effect of intersolution interval, chlordiazepoxide, and amphetamine on anticipatory contrast
}

\author{
CHARLES F. FLAHERTY and GRACE A. ROWAN \\ Rutgers University, New Brunswick, New Jersey
}

\begin{abstract}
Previous experiments have shown that the intake of a $0.15 \%$ saccharin solution is suppressed if saccharin access is followed by access to $32 \%$ sucrose in brief daily pairings. The present experiments found that: (1) the degree of suppression was not altered when no time elapsed between presentation of the two solutions each day ( $15 \mathrm{sec}$ had been the minimum in previous experiments and was used as the control in this experiment); (2) the degree of suppression was not altered by chlordiazepoxide $(6,12$, or $20 \mathrm{mg} / \mathrm{kg})$, although the drug had large appetite-stimulating effects; (3) suppression was not influenced by amphetamine $(0.25$ or $0.50 \mathrm{mg} / \mathrm{kg}$ ); and (4) contrast could be established or eliminated, even after extended training, by manipulating the sequences of solutions presented (saccharin-saccharin or saccharin-sucrose). The results were interpreted in terms of a contrast effect based on the learned anticipation of a preferred substance. The chlordiazepoxide data suggest that this contrast is different from successive negative contrast, and the intersolution interval data suggest that the occurrence of contrast rather than a reinforcement effect is not due to a time gap between presentations of the two solutions.
\end{abstract}

If rats are given single daily pairings of an access period to a $0.15 \%$ saccharin solution followed by an access period to a $32 \%$ sucrose solution, the intake of the saccharin solution is suppressed. This suppression occurs relative to control conditions, which have included access to the saccharin alone, access to saccharin followed by access to the same saccharin solution again, and access to saccharin followed by access to a $2 \%$ sucrose solution (Flaherty \& Checke, 1982; Flaherty \& Rowan, 1985). This suppression of saccharin intake has been interpreted in terms of a contrast effect based on relative preferences for the first and second substances (e.g., Flaherty \& Rowan, 1985).

The occurrence of a contrast effect, rather than a reinforcement effect (an increase in saccharin consumption when it is followed by access to $32 \%$ sucrose), is surprising. The present study further analyzed this contrast effect in terms of (1) the influence of the intersolution interval on the degree of saccharin suppression (Experiment 1); (2) the influence of chlordiazepoxide (administration of which will eliminate successive negative contrast effects) on saccharin suppression (Experiment 2); and (3) the influence of chlordiazepoxide on the reversal of contrast when the solution pairings are reversed (Experiment 3).

\section{EXPERIMENT 1}

In previous experiments, the length of the interval between presentations of two solutions influenced the degree

This research was supported by grants from the National Institute of Mental Health (MH40489) and the Charles and Johanna Busch Memorial Fund. The assistance of Kelvin Yu is appreciated. Correspondence should be addressed to Charles Flaherty, Psychology Department, Busch Campus, Rutgers University, New Brunswick, NJ 08903. of suppression that occurred-as the interval increased in the range of $1 \mathrm{~min}$ to $30 \mathrm{~min}$, the degree of suppression decreased (Flaherty \& Checke, 1982). In other experiments, a substantial degree of suppression occurred when the interbottle interval was only $15 \mathrm{sec}$ (Flaherty \& Rowan, 1985, 1986), the minimum interbottle interval used in earlier experiments. The duration of this interval might have been related to the fact that the availability of sucrose decreased rather than increased saccharin intake. That is, the animals were required to lick the saccharin tube (a minimum of 1 lick was required to initiate timing of a 3-min access period) in order to obtain sucrose from the second tube. However, the passage of the 15 -sec interval between tubes might have been sufficient to degrade the contingent relationship between licking on the first tube and gaining access to the second tube, and, in the absence of a contingency, a contrast effect rather than a reinforcement effect occurred.

In the present experiment, we examined the effect on degree of contrast of a 0 -sec interbottle interval (in which the second tube was immediately inserted following the retraction of the first tube) versus a 15 -sec interbottle interval. Thus, separate groups of animals were given either saccharin followed by saccharin or saccharin followed by sucrose, with either a $15-\mathrm{sec}$ or a 0 -sec interbottle interval.

\section{Method}

Subjects. The subjects were 20 male Sprague-Dawley-derived rats purchased from Blue Spruce. The rats were approximately 110 days old and weighed between 375 and $475 \mathrm{~g}$ at the start of the experiment. They were maintained at $82 \%$ of their free-feeding weights, housed individually with water continuously available, and kept on a 14:10-h light:dark cycle.

Apparatus. Testing was conducted in three Plexiglas chambers measuring $30 \times 25 \times 25 \mathrm{~cm}$. Two holes, $1.5 \mathrm{~cm}$ in diameter, were located $21.7 \mathrm{~cm}$ apart on one wall of the apparatus, $4 \mathrm{~cm}$ above 
the hardware-cloth floor. Solutions were delivered via graduated cylinders with metal spouts. The cylinders were attached to motors that advanced the spouts or withdrew them from the access holes. Licks were recorded through a contact relay circuit and microprocessors.

Procedure. The experiment was designed as a $2 \times 2$ factorial, varying the solution available in the second tube $(0.15 \%$ saccharin or $32 \%$ sucrose) and the interval between tubes ( 15 or $0 \mathrm{sec})$. Five animals were randomly assigned to each group. All animals received the $0.15 \%$ saccharin solution in the first tube, which was available for a 3-min period beginning with the first lick. The second tube was available for a 5-min period beginning with the first lick. Neither tube was available when the animals were first placed in the chamber. The first tube was presented approximately 30-45 sec after the animals were placed in the boxes.

The animals received one pairing of the two solutions each day for 14 days. The number of licks to each tube was recorded. The saccharin solution was prepared on a weight/volume basis and the sucrose solution was prepared by weight (sucrose/[sucrose + water]).

\section{Results}

The mean lick frequencies obtained in the various conditions across the acquisition period are presented in Figure 1. It is clear that saccharin intake was suppressed in the animals that subsequently received access to sucrose $[F(1,16)=32.00, p<.05]$. This suppression developed across the acquisition period [solution $\times$ block: $F(6,92)$ $=10.27, p<.05]$ and was reliable by the second block [least significant difference $(L S D)$ test, $p<.05$ ]. There was no difference in the development of suppression as a function of the intersolution interval (all $F \mathrm{~s}<1.00$ ).

Analysis of the lick frequencies obtained on the second bottle available each day showed that the animals licked substantially more for the sucrose solution (mean licks $=1,434.6$ ) than for the saccharin solution (mean licks $=468.1)$. This difference was highly reliable $[F(1,16)=80.48, p<.05]$ and did not vary across blocks $(F<1.00)$.

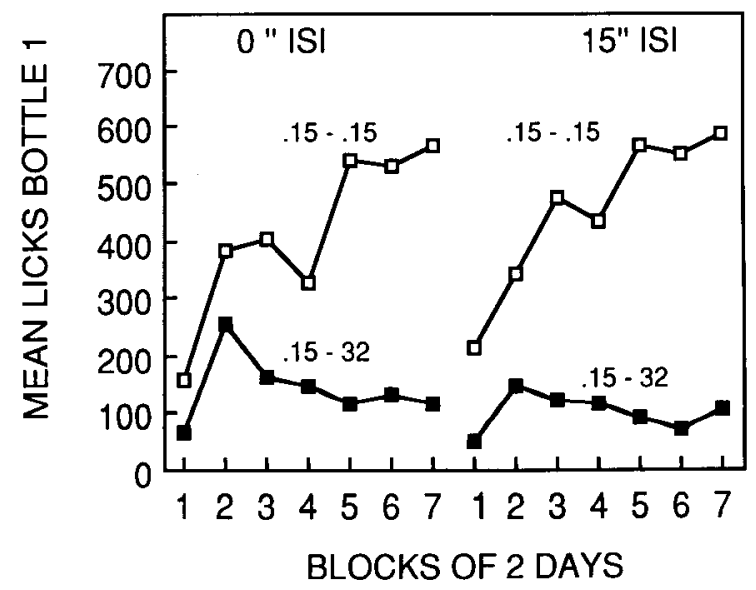

Figure 1. The mean lick frequency on the first bottle available as a function of solution condition and as a function of interbottle interval. The .15-32 condition refers to a $0.15 \%$ saccharin solution in the first bottle available and a 32\% sucrose solution in the second bottle available. The $.15-.15$ condition indicates the saccharin solution in both bottles. ISI = intersolution interval.

\section{Discussion}

These results support those obtained in previous experiments in showing that the animals that received the sequence $0.15 \%$ saccharin- $32 \%$ sucrose consumed less of the initial saccharin solution than the animals that received the sequence $0.15 \%$ saccharin- $0.15 \%$ saccharin. We interpret this pattern of results as indicating a contrast effect based on the learned anticipation of the second solution. That is, we believe that the animals learned the contingent relationship between the two solutions (Rescorla, 1968, 1978) and, once this learning was completed, the sampling of the first substance (saccharin) served as a cue for the second substance (either saccharin or sucrose). The signaling of a preferred second substance (sucrose) might have served to devalue the saccharin and/or to elicit behaviors that competed with consumption of the saccharin. The results of the present experiment showed that the degree of contrast, and presumably the associative structure supporting the contrast, was not sensitive to variations in the intersolution interval, which ranged from 0 to $15 \mathrm{sec}$, whereas previous experiments have shown that intervals longer than several min did reduce contrast (Flaherty \& Checke, 1982).

A question arises regarding the relationship of anticipatory contrast to successive negative contrast. In the successive contrast procedure, rats are typically given access to $32 \%$ sucrose for 5 min per day for 10 days and then shifted to $4 \%$ sucrose. Animals so shifted drink considerably less of the $4 \%$ sucrose than do unshifted controls, but eventually recover over a 3- or 4-day postshift period. Successive contrast effects also occur when the concentration of saccharin solutions is shifted (Flaherty \& Rowan, 1986) and when rats are shifted from sucrose to saccharin (unpublished data from our laboratory).

The successive contrast effects that occur when rats are shifted from $32 \%$ to $4 \%$ sucrose are reliably reduced by anxiolytic drugs such as ethanol, chlordiazepoxide (CDP), sodium amobarbital, morphine, and midazolam (Becker, 1986; Becker \& Flaherty, 1982, 1983; Flaherty \& Driscoll, 1980; Flaherty, Lombardi, Wrightson, \& Deptula, 1980; Rowan \& Flaherty, 1987). However, another form of contrast, simultaneous contrast, which occurs when rats are rapidly and regularly shifted between sucrose solutions within a day, is apparently not influenced by anxiolytic drugs (e.g., Flaherty, Becker, \& Driscoll, 1982; Flaherty, Lombardi, Kapust, \& D'Amato, 1977). This pattern of results has led to the suggestion that an emotional response is causally involved in successive negative contrast, but not in simultaneous contrast (e.g., Flaherty, Becker, \& Pohorecky, 1985; Flaherty \& Rowan, 1986).

The next experiment investigated the effects of an anxiolytic and a psychomotor stimulant on anticipatory contrast.

\section{EXPERIMENT 2}

The subjects were those used in Experiment 1, maintained under the same conditions except for the drug treatment. CDP and amphetamine were used. CDP has been 
shown to reduce both negative contrast in runway behavior (Rosen \& Tessel, 1970) and behavioral contrast (Baltzer, Huber, \& Weiskrantz, 1979), as well as contrast in consummatory behavior. Amphetamine, a stimulant normally thought of as having appetite-suppressing effects, has recently been reported to have appetite-stimulating effects in low doses (e.g., Hoebel, Hernandez, Monaco, \& Miller, 1981; Jain, Kyriakides, Silverstone, \& Turner, 1980). Furthermore, amphetamine, an agent that potentiates the effectiveness of endogenous dopamine, has been reported to enhance the rewarding effects of brain stimulation (Wise, 1982). Because dopamine has been postulated to underlie at least some aspects of the neural correlates of reward (Fibiger \& Phillips, 1979), it is possible that amphetamine may influence the degree of reward contrast.

\section{Method}

Subjects. The rats used in Experiment 1 served as subjects.

Apparatus. The apparatus was the same as that used in Experiment 1.

Procedure. The animals were maintained under the same conditions as in Experiment 1. In addition, the effects of CDP and amphetamine were investigated using a within-subjects procedure. Each drug dose was evaluated by using the following 4-day cycle: Day 1, saline; Day 2, drug; Day 3, saline; Day 4, not run. The drugs and doses tested were, in order of testing, 6,12 , and $20 \mathrm{mg} / \mathrm{kg}$ of CDP, and 0.25 and $0.50 \mathrm{mg} / \mathrm{kg}$ of amphetamine. Both drugs were administered intraperitoneally, the CDP $30 \mathrm{~min}$ prior to the session and the amphetamine 20 min prior to the session.

\section{Results}

Because the interbottle interval factor $(15 \mathrm{sec} v \mathrm{vs} .0 \mathrm{sec})$ influenced neither contrast nor the effects of the drugs, the data from the two conditions were combined for presentation. The mean lick frequency obtained under the various drug conditions is presented in Figure 2. The CDP had a clear appetite-stimulating effect, increasing the lick

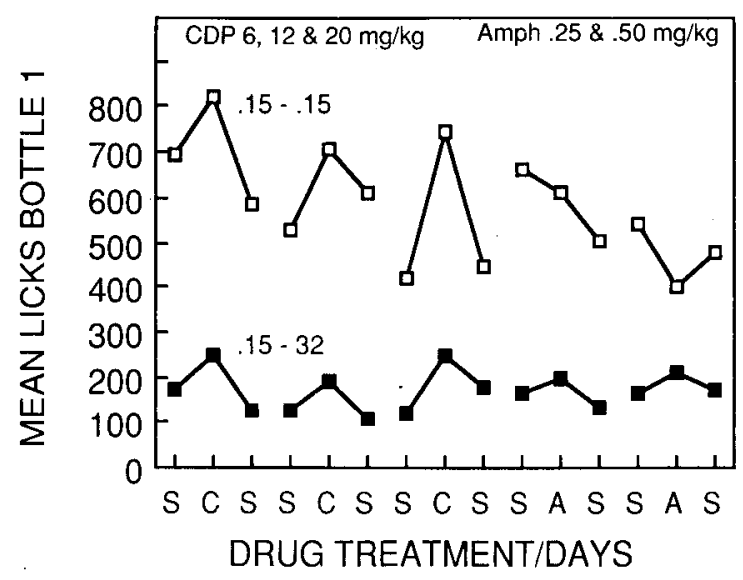

Figure 2. The effect of chlordiazepoxide (CDP) and amphetamine (Amph) on lick frequency in the two solution conditions. Injections were given only on the 2 nd day of each 3-day cycle. The data points in this figure continue from those in Figure 1. frequency of both contrast and control groups, but it did not influence the degree of contrast.

Each CDP dose was analyzed separately, and all showed the same statistical effect: an elevation in lick frequency in both contrast (.15-32) and control (.15-.15) groups on CDP days as compared with saline days $[6 \mathrm{mg} / \mathrm{kg}: F(2,30)$ $=12.26, p<.05 ; 12 \mathrm{mg} / \mathrm{kg}: F(2,32)=6.64, p<.05$; $24 \mathrm{mg} / \mathrm{kg}: F(2,32)=11.09, p<.05]$. Contrast remained reliable throughout the CDP treatment phase [6 mg/kg: $F(1,16)=60.29, p<.05 ; 12 \mathrm{mg} / \mathrm{kg}: F(1,16)$ $=57.54, p<.05 ; 20 \mathrm{mg} / \mathrm{kg}: F(1,16)=21.10$, $p<.05]$ and there was no indication that the degree of contrast was reduced by the drug $(F<1.00$ for all group $X$ day interactions).

The effects obtained with amphetamine (also shown in Figure 2) fall into a pattern different from that obtained with CDP. Rather than increasing intake, amphetamine had a tendency to decrease intake of the saccharin solution. In the case of the $0.25 \mathrm{mg} / \mathrm{kg}$ dose of amphetamine, there was a clear decrement in lick frequency across the 3-day cycle, with Day 3 (the day after the amphetamine injection) being the lowest of the 3 days $[F(2,32)=4.66$, $p<.05]$. No statistically reliable effects were obtained with the $0.50 \mathrm{mg} / \mathrm{kg}$ dose, but there was a clear tendency for the amphetamine to decrease licking in the .15-.15 group [group $\times$ day, $F(2,30)=3.13, p<.06$ ]. A reliable contrast effect was maintained across both amphetamine cycles $[F(1,16)=27.50, p<.05$ and $F(1,16)=$ $10.88, p<.05]$. A comparison between the 2 days on which amphetamine was injected indicated that a reliable contrast occurred on both injection days [group $\times$ day, $F(1,16)=6.18, p<.03$; followed by $L S D$ tests], but contrast was smaller when the higher dose of amphetamine was administered ( $L S D$ test), due to a decline in lick frequency in the .15-.15 group (reliable by $L S D$ test). There was no reliable change in the contrast group (.15-32).

Lick frequency on the second tube available to the animals was also measured, but, because the drugs did not have pronounced effects and because the data did not depart substantially from the results obtained in Experiment 1 , these results will not be presented.

\section{Discussion}

CDP and other anxiolytic drugs have been found to release behavior suppressed by a variety of environmental contingencies, including novelty, punishment, nonreward, and aversive stimuli (e.g., File, 1985; Gray, 1982; Treit, 1985). CDP also has a dose-dependent effect that reduces the degree of contrast that occurs when animals are shifted from $32 \%$ to $4 \%$ sucrose solutions, with doses of 6 or $8 \mathrm{mg} / \mathrm{kg}$ sufficing to eliminate contrast (Becker \& Flaherty, 1983; Flaherty et al., 1980). The failure of CDP to influence contrast in the present experiment (although it did have an appetite-stimulating effect, as found in other studies, e.g., Cooper \& Estall, 1985; Riley \& Lovely, 1978) suggests that anticipatory 
contrast and successive contrast may be mediated by different mechanisms. Such a possibility is also suggested by the procedural differences between the two paradigms. In the successive contrast paradigm, the animals' preferred substance is replaced by a less preferred substance-a situation that would be expected to lead to an analogue of disappointment. On the other hand, in the anticipatory contrast paradigm, the animals are responding to a substance that signals the impending occurrence of a preferred solution-a situation that should not be "disappointing."

Thus, although consummatory behavior is suppressed in both situations, it is likely that the suppression occurs for different reasons. This interpretation is supported by a previous experiment, which showed that the suppression that occurred in anticipatory contrast was related to the impending $32 \%$ sucrose each day rather than to the $32 \%$ sucrose solution received on the previous day (Flaherty \& Rowan, 1985). These data are reminiscent of some results obtained in behavioral contrast by Williams (1979). He found that the terminal schedule in a threeply multiple schedule exerted more control over responding in the middle schedule than did the initial schedule.

Amphetamine was included to test the possibility that a stimulant drug (and dopamine agonist) might have effects when a tranquilizer did not. The results show quite clearly that amphetamine did not influence contrast.

\section{EXPERIMENT 3}

The failure of CDP and amphetamine to influence contrast might be due to the fact that the mechanism of action of these drugs is not related to the processes that produce anticipatory contrast (as suggested above). Alternatively, it is possible that anticipatory contrast, once established, is simply resistant to change. The present experiment examined the flexibility of consummatory behavior in this contrast situation by reversing the solution contingencies in effect for the animals used in Experiments 1 and 2 ; that is, the animals that had received $0.15 \%$ saccharin in the first tube and $32 \%$ sucrose in the second tube were shifted to the .15-.15 condition, and the animals that had received the $.15-.15$ condition were shifted to the $.15-32$ condition. The interbottle interval conditions $(0$ vs. $15 \mathrm{sec}$ ) were left unchanged.

\section{Method}

Subjects. The subjects were the same as those used in the previous experiments.

Apparatus. The apparatus was the same as that used in the previous experiments.

Procedure. The procedure was the same as that used in Experiment 1 , except that the saccharin-sucrose relationships between groups were reversed; that is, the animals that had received the $.15-.15$ condition were shifted to the $.15-32$ condition, and vice versa. The interbottle interval conditions were not changed. These conditions were maintained for 10 days.

\section{Results}

The intake data for the first bottle are presented in Figure 3. The shift in the saccharin-sucrose relationships

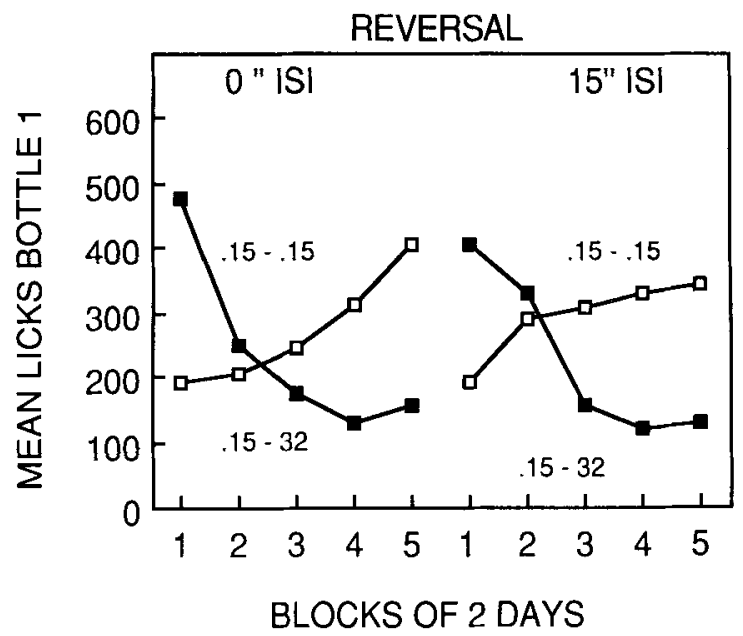

Figure 3. The effect of reversing the solution pairings on lick frequency. The group labeled .15-32 was shifted from the .15-.15 condition, and vice-versa. The data in this figure are continuous with those in Figure 2. ISI = intersolution interval.

clearly led to a shift in consummatory behavior. The rats that were shifted from the .15-32 condition to the .15-.15 condition showed an increase in lick frequency, and those that were shifted from the .15-.15 condition to the .15-32 condition showed a decrease in lick frequency.

Analysis of the data indicated a reliable solution $\times$ block effect $[F(4,64)=24.55, p<.05]$ and no reliable effects of interbottle interval $(F \mathrm{~s}<1.00)$. Subsequent analysis of the solution $\times$ block term with the $L S D$ test showed that the .15-32 group licked more than the $.15-.15$ group in Block 1, but less than the .15-.15 group in Blocks 4 and 5 . Both the increase in lick frequency found in the group switched to the $.15-.15$ condition and the decrease in lick frequency found in the group switched to the $.15-32$ condition were reliable by $L S D$ test.

Intake data on the second bottle available to each group showed that the animals given access to the $32 \%$ sucrose solution licked substantially more frequently (mean licks $=1,478.9$ ) than the animals given access to the $0.15 \%$ saccharin solution (mean licks $=422)[F(1,16)=123.80$, $p<.05]$.

\section{Discussion}

Intake of the initial $0.15 \%$ saccharin solution was under the control of the solution that followed it. When the second tube was switched from $0.15 \%$ saccharin to $32 \%$ sucrose, the lick frequency on the first tube abruptly declined. Similarly, when the second tube was switched from $32 \%$ sucrose to $0.15 \%$ saccharin, the intake of the $0.15 \%$ saccharin available in the first tube increased.

The rapid changes in consummatory behavior that occurred in this experiment suggest that the failure of the drugs in Experiment 2 to produce effects was not due to behavioral inflexibility on the part of the animals. These results further suggest that the associative process, if that is what controlled the lick behavior to the first tube, was itself subject to rapid change following changes in the environmental circumstances. 


\section{GENERAL DISCUSSION}

These experiments showed that the intake of the initial $0.15 \%$ saccharin solution was suppressed by the subsequent availability of $32 \%$ sucrose and that this contrast effect was not influenced by CDP or amphetamine, nor was it altered when there was no time lag between the retraction of the saccharin tube and the presentation of the sucrose tube. The results obtained with CDP in Experiment 2 supported an earlier suggestion (Flaherty \& Rowan, 1985) that anticipatory contrast was not a successive negative-contrast effect based on the comparison of the $0.15 \%$ saccharin with the memory of the $32 \%$ sucrose solution received on the previous day. The earlier argument was grounded on procedural details and on the effects of the within-subjects manipulation of the saccharin-sucrose pairings. Experiment 2 showed that CDP, in doses of 6,12 , and $20 \mathrm{mg} / \mathrm{kg}$, had no effect at all on anticipatory contrast, whereas other studies showed that doses of 6 and $8 \mathrm{mg} / \mathrm{kg}$ completely eliminated successive negative contrast (Becker \& Flaherty, 1983; Flaherty et al., 1980). It is important to note that the failure of the drug to reduce anticipatory contrast cannot be related to a general failure of the drug to affect behavior in these circumstances, because CDP did have a powerful appetitestimulating effect (however, it influenced control and contrast animals equally). Also, Experiment 3 showed that the lack of effectiveness of the drug could not be attributed to an insensitivity of the licking response because of overtraining - the licking behavior of both contrast and control groups changed rapidly when the saccharin-sucrose contingencies were reversed.

The results obtained in these experiments do not alter the interpretation of the suppression of saccharin intake described earlier (Experiment 1) and in previous studies. That is, the suppression reflects a contrast effect based on the learned anticipation of a preferred substance. This anticipation devalues the saccharin solution and/or leads to competing behaviors that reduce intake of the saccharin. Competing responses based on spatial location cannot completely explain anticipatory contrast, because it has been obtained when both solutions were presented through the same opening (Flaherty \& Checke, 1982, Experiment 3 ), and behavioral contrast, aspects of which may be closely related to anticipatory contrast, occurs regularly with a single manipulandum (Williams, 1981, 1983). Thus, devaluation of the saccharin solution, perhaps based on Pavlovian conditioned representational processes (Colwill \& Rescorla, 1985; Holland \& Straub, 1979), may play a major role in the occurrence of anticipatory contrast. The CDP data suggest that this anticipation is not mediated by an aversive emotional state, as may be the case in the occurrence of successive negative contrast.

The occurrence of the contrast effect, rather than a reinforcement effect, with the 0-sec interbottle interval condition suggests that the contrast was not due to a degraded instrumental contingency produced by the presence of a time interval between the presentation of the two tubes. Recently gathered data do show, however, that the imposition of a specific instrumental contingency, in the form of a required lick count, eliminates contrast and leads to a reinforcement effect in terms of the latency to lick the first tube (i.e., the .15-32 animals are faster than the $.15-.15$ animals, Flaherty \& Grigson, in press). Thus, in the absence of a strong instrumental contingency, the behavior of the rats in this situation may be controlled by Pavlovian associative processes and contrast effects based on the comparison of predictor and predicted substances that differ in hedonic value.

\section{REFERENCES}

Baltzer, V., Huber, H., \& Weiskrantz, L. (1979). Effects of various drugs on behavioral contrast using a double-crossover procedure. Behavioral \& Neural Biology, 27, 330-341.

BECKER, H. C. (1986). Comparison of the effects of the benzodiazepine midazolam and three serotonin antagonists on a consummatory conflict paradigm. Pharmacology, Biochemistry \& Behavior, 24, 1057-1064.

Becker, H. C., \& Flaherty, C. F. (1982). Influence of ethanol on contrast in consummatory behavior. Psychopharmacology, 77 253-258.

Becker, H. C., \& Flaherty, C. F. (1983). Chlordiazepoxide and ethanol additively reduce gustatory negative contrast. Psychopharmacology, 80, 35-37.

CoOper, S. J., \& EsTALL, L. (1985). Behavioral pharmacology of food, water and salt intake in relation to drug actions at benzodiazepine receptors. Neuroscience \& Biobehavioral Reviews, 9, 5-19.

Colwill, R. M., \& Rescorla, R. A. (1985). Instrumental responding remains sensitive to reinforcer devaluation after extensive training. Journal of Experimental Psychology: Animal Behavior Processes, 11, 520-536.

Fibiger, H., \& Phillips, A. (1979). Dopamine and the neural mechanisms of reinforcement. In A. S. Horn, J. Korf, \& B. Westerink (Eds.), The neurobiology of dopamine (pp. 597-615). London: Academic Press.

FILE, S. (1985). Animal models for predicting clinical efficacy of anxiolytic drugs: Social behavior. Neuropsychobiology, 13, 55-62.

FlaherTy, C. F. (1982). Incentive contrast: A review of behavioral changes following shifts in reward. Animal Learning \& Behavior, 10, 409-440.

Flaherty, C. F., Becker, H. C., \& Driscoll, C. (1982). Conditions under which amobarbital sodium influences consummatory contrast. Physiological Psychology, 10, 122-128.

Flaherty, C. F., Becker, H. C., \& Pohorecky, L. (1985). Correlation of corticosterone elevations and negative contrast varies as a function of postshift day. Animal Learning \& Behavior, 13, 309-314.

Flaherty, C. F., \& CheCKe, S. (1982). Anticipation of incentive gain. Animal Learning \& Behavior, 10, 177-182.

Flaherty, C. F., \& Driscoll, C. (1980). Amobarbital sodium reduces successive gustatory contrast. Psychopharmacology, 69, 161-162.

Flaherty, C. F., \& Grigson, P. S. (in press). From contrast to reinforcement: Role of response contingency in anticipatory contrast. Journal of Experimental Psychology: Animal Behavior Processes.

Flaherty, C. F., Lombard, B., Kapust, J., \& D'Amato, M. (1977). Incentive contrast undiminished by extended testing, imipramine, or chlordiazepoxide. Pharmacology, Biochemistry \& Behavior, 7, 315-322.

Flaherty, C. F., Lombard, B. R., Wrightson, J., \& Deptula, D. (1980). Conditions under which chlordiazepoxide influences successive gustatory contrast. Psychopharmacology, 67, 269-277.

Flaherty, C. F., \& Rowan, G. A. (1985). Anticipatory contrast: Within subjects analysis. Animal Learning \& Behavior, 13, 2-5. 
Flaherty, C. F., \& Rowan, G. A. (1986). Successive, simultaneous and anticipatory contrast in the consumption of saccharin solutions. Journal of Experimental Psychology: Animal Behavior Processes, 12, 381-393.

GraY, J. A. (1982). The neuropsychology of anxiety. Oxford: Oxford University Press.

Hoebel, B., Hernandez, L., Monaco, A., \& Miller, W. (1981). Amphetamine-induced overeating and overweight in rats. Life Sciences, 28, 77-82.

Holland, P. C., \& Straub, J. J. (1979). Differential effects of two ways of devaluing the unconditioned stimulus after Pavlovian appetitive conditioning. Journal of Experimental Psychology: Animal Behavior Processes, 5, 65-78.

Jain, S., Kyriakides, M., Silverstone, T., \& Turner, P. (1980). The effect of small and moderate doses of d-amphetamine on hunger, mood and arousal in man. Psychopharmacology, 70, 109-111.

RESCORLA, R. A. (1968). Probability of shock in the presence and absence of CS in fear conditioning. Joumal of Comparative \& Physiological Psychology, 66, 1-5.

RESCORLA, R. A. (1978). Some implications of a cognitive perspective on Pavlovian conditioning. In S. H. Hulse, H. Fowler, \& W. K. Honig (Eds.), Cognitive processes in animal behavior (pp. 15-50). Hillsdale, NJ: Erlbaum.
Riley, A., \& Lovely, R. (1978). Chlordiazepoxide-induced reversal of an amphetamine-established aversion: Dipsogenic effects. Physiological Psychology, 6, 488-492.

Rosen, A., \& Tessel, R. (1970). Chlorpromazine, chlordiazepoxide and incentive shift performance in the rat. Journal of Comparative \& Physiological Psychology, 72, 257-262.

Rowan, G. A., \& Flaherty, C. F. (1987). The effects of morphine in the consummatory contrast paradigm. Psychopharmacology, 93, 51-58.

TREIT, D. (1985). Animal models for the study of anti-anxiety agents: A review. Neuroscience \& Biobehavioral Reviews, 9, 203-222.

Williams, B. A. (1979). Contrast, component duration, and the following schedule of reinforcement. Journal of Experimental Psychology: Animal Behavior Processes, 5, 379-396.

Williams, B. A. (1981). The following schedule of reinforcement as a fundamental determinant of steady-state contrast in multiple schedules. Journal of the Experimental Analysis of Behavior, 35, 293-310.

Williams, B. A. (1983). Another look at contrast in multiple schedules. Journal of the Experimental Analysis of Behavior, 39, 345-384. WISE, R. H. (1982). Common neural basis for brain stimulation reward, drug reward, and food reward. In B. Hoebel \& D. Novin (Eds.), Neural basis of feeding and reward (pp. 445-454). Brunswick, ME: Haer Institute.

(Manuscript received June 11, 1987;

revision accepted for publication September 22, 1987.) 